\title{
REVIEW
}

\section{The Problems with Polio: Toward Eradication}

Gemma Lien • David L. Heymann

To view enhanced content go to www.infectiousdiseases-open.com

Received: May 28, 2013 / Published online: September 17, 2013

(c) The Author(s) 2013. This article is published with open access at Springerlink.com

\section{ABSTRACT}

The global health effort to eradicate poliomyelitis (polio) has encountered a number of unforeseen and unpredictable challenges. This article provides a timely review of progress made toward eradication, including the polio vaccines in use, and explores the reasons for delays in eradication target dates. It provides an overview of some of the remaining barriers to eradication and looks toward overcoming these through the Polio Eradication and Endgame Strategic Plan.

Keywords: Polio; Polio eradication; Polio endgame; Polio vaccine; Poliomyelitis; Poliovirus

G. Lien $(\bowtie) \cdot$ D. L. Heymann

Public Health England, Wellington House, 133-155

Waterloo Road, London SE1 8UG, UK

e-mail: gemma.lien@phe.gov.uk

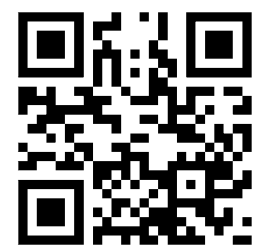

Enhanced content for this article is

available on the journal web site:

www.infectiousdiseases-open.com

\section{INTRODUCTION}

The global health effort to eradicate poliomyelitis (polio) has encountered a number of unforeseen and unpredictable challenges which have been well documented [1]. This article provides a timely review of these challenges and looks toward overcoming the remaining barriers to eradication.

\section{METHODS}

The authors undertook a comprehensive literature review using the Internet and the databases JSTOR, PubMed, ScienceDirect and SwetsWise. The following search terms were used: "polio", "poliomyelitis", "polio eradication", "polio endgame", "polio vaccine" and "poliovirus".

\section{DISCUSSION}

Polio is a highly infectious viral disease, which can cause paralysis and, in some cases, death. Wild polioviruses are those that occur naturally. There are three serotypes of wild poliovirus: type-1, type-2, and type-3. The poliovirus enters 
the body through the mouth, multiplies in the oropharynx and the small intestine and exits in the feces from which it can spread rapidly through a community, especially in areas with poor hygiene and sanitation. The virus invades the local lymphoid tissues in the gastrointestinal tract, and may then enter the bloodstream and spread to the central nervous system. The virus may also spread to the central nervous system along the peripheral nerves. Over $90 \%$ of people infected with poliovirus have either no or very mild symptoms, which can easily go unrecognized [2]. This makes it very difficult to identify an outbreak immediately as asymptomatic infections can spread the infection 'silently' to others before the first case of polio paralysis is detected. Therefore, herd immunity must be attained to prevent transmission and outbreaks of polio occurring.

Before the twentieth century, poor hygiene and sanitation meant that almost all children were exposed to poliovirus during infancy, which enabled natural immunity to build up in populations. The industrial revolution brought great sanitary improvements, including the separation of sewage from drinking water. While this proved vital in increasing public health standards in general, it initially had disastrous effects in relation to polio cases. It reduced childhood exposure to the virus and lowered immunity levels in communities, creating the perfect setting for epidemics to ignite [3].

By the late 1980s, polio had been eliminated from most industrialized countries by routine immunization programs. However, it was estimated that polio still paralyzed more than 1,000 children every day globally, and that the poliovirus was circulating in more than 125 lesser developed countries [4]. Building on the global health success of the eradication of smallpox, and encouraged by the progress made toward interrupting wild poliovirus transmission in the Americas in the early 1980s, in 1988 the World Health Assembly declared the commitment of the World Health Organization (WHO) to the global eradication of poliomyelitis by the year 2000 [5]. The Global Polio Eradication Initiative (GPEI) was formed to achieve this target, led by WHO, the United Nations Children's Fund, Rotary International, and the United States Centers for Disease Control and Prevention [6].

The GPEI understood that increasing population immunity by routine vaccination supplemented by national mass immunization campaigns, and enhancing the epidemiological surveillance of the disease so that outbreaks could be rapidly detected and contained, would be the key to interrupting the transmission of wild poliovirus and achieving eradication [6].

The vaccine most used globally is the trivalent oral polio vaccine (tOPV or 'Sabin vaccine'), which is effective against all three types of wild poliovirus. Use of tOPV can result in the 'passive' immunization of people living in areas of poor hygiene and sanitation who have not been directly vaccinated, as the virus continues to be excreted through the feces into the environment for several weeks after vaccination. A further advantage to its use is its cost, estimated to be between 11 and 14 US cents per dose [7].

There are also two more oral polio vaccines in use today: the monovalent vaccine (mOPV) and the bivalent vaccine (bOPV). In children being immunized for the first time, the monovalent vaccine (mOPV), consisting of just one type of the live attenuated strains of poliovirus, provides a greater immunity to the specific type of poliovirus being targeted and also provides increased immunity for the same number of doses compared with tOPV. This 
may be because there is no competition from the other two virus types in the vaccine [8]. The bivalent vaccine (bOPV) consists of live attenuated strains of both type- 1 and type- 3 poliovirus and improves the efficiency and impact of vaccination campaigns in areas where both types of poliovirus co-circulate. It is more effective than tOPV and almost as effective as mOPV in achieving protection [9].

Unfortunately, in very rare cases, (approximately 1 in every 2.7 million first doses of the vaccine), the oral polio vaccines can cause a condition known as vaccineassociated paralytic polio [7]. Even more concerning is the potential for the live attenuated strains of the vaccine viruses to revert and re-acquire neurovirulence, resulting in circulating vaccine-derived polioviruses (cVDPVs) [10]. cVDPVs could pose a threat in a post-eradication world, with the ability to cause devastating outbreaks of polio at a time when immunity levels are reduced.

In most high-income countries, where the risk of polio infection is low, the inactivated polio vaccine (IPV or 'Salk vaccine') is used. IPV consists of "killed" strains of all three polioviruses, which is delivered via an injection. As it is not a "live" vaccine, IPV poses no risk to the recipient of vaccineassociated paralytic polio, nor is there any possibility of cVDPVs emerging [11]. However, it does need to be administered by a trained health worker, induces very low levels of immunity in the intestine and is over five times more expensive than the oral polio vaccine [11].

Following its launch in 1988, the GPEI had a promising start and the Americas was the first WHO Region to be certified polio-free of all three types of wild poliovirus in 1994. By the year 2000, the global incidence of polio had been reduced by over 99\% [12] and every endemic country had implemented some form of polio-eradication strategy. Much effort had been made to attain herd immunity by supplementing the preceeding level of polio vaccination coverage in routine immunization programs with compaings [13].

However, delays in the global implementation of eradication strategies, in part due to lack of political commitment, funding and competing development and health priorities meant that the initial target for eradication by the year 2000 was missed. Nevertheless, progress continued with the certification of two more WHO Regions as polio-free: the Western Pacific Region in 2000 [14] and the European Region in 2002 [15].

In 2003, only six polio-endemic countries remained: Afghanistan, Egypt, India, Niger, Nigeria and Pakistan. Although Egypt and Niger were later declared polio-free by 2005, the remaining four countries faced various challenges to the eradication effort over the next 10 years. Following the elimination of type-2 wild poliovirus from human populations in 1999 when the last infection was identified in India [16], and because tOPV provides less optimum protection against poliovirus serotypes 1 and 3 in some tropical settings, the monovalent and bivalent formulations of the vaccine were introduced to more closely target and rapidly interrupt the remaining virus types in circulation, particularly in densely populated areas of high intensity of transmission [17].

India's greatest challenge to eradication was the sub-optimal effectiveness of tOPV in areas of high birth rates, poor sanitation as well as dense and migratory communities. This was particularly apparent in northern India and was only overcome by a substantial effort to push coverage rates to over 95\% in particularly vulnerable populations and areas, and the 
careful and tactical use of mOPV and bOPV [1]. India was finally removed from the WHO list of polio-endemic countries in early 2012; an enormous achievement, considering that in 2009 , India had the highest number of polio cases in the world [18]. It is expected that India will be officially certified as polio-free in 2014 [19].

The nature of poliovirus has posed its own challenge to eradication. Every child needs to be vaccinated multiple times to ensure full immunity, depending on the vaccine used [20]. This provides a significant logistical challenge to vaccinators, especially with migratory, displaced or hard to access populations. It can be very difficult to ascertain when and how many doses of vaccine each child has received and how many children were missed on vaccination days [1]. This can pose a high risk to immunity levels as the virus may be transmitted over large distances with little warning.

Natural disasters such as floods, earthquakes, hurricanes and tsunamis can also contribute to delays in eradication efforts. These can all have a detrimental impact on communications and road and health infrastructures, in some cases making it impossible to reach people except by air. Hospitals, medical centers and cold chain storage facilities can be damaged or destroyed and local health workers displaced.

The re-importation of wild poliovirus to countries that were previously polio-free has also complicated efforts. Angola, Chad and the Democratic Republic of Congo have all experienced re-established transmission, resulting in reservoirs from where neighboring countries have been repeatedly infected. In addition, the transmission of cVDPVs has also caused problems in a number of countries. Poor management and oversight of polio and routine immunization campaigns continue to be a major risk factor for outbreaks following reimportation of the poliovirus into previously polio-free countries [1]. Gaps in the quality of acute flaccid paralysis surveillance have also compromised the speed of outbreak response activities.

Only three polio-endemic countries remain in 2013: Afghanistan, Nigeria and Pakistan. It can be argued that geopolitical events in all three countries, such as war and insecurity, in addition to the loss of community confidence in the immunization program in some areas of these countries, have continued to hamper eradication progress. Civil disturbance displaces children and can result in the blocking of access routes during vaccination campaigns. Deep distrust of perceived Westernled initiatives has also impacted on polio immunization efforts. False rumors, such as those that circulated in Nigeria in 2003 that the polio vaccine was being used to sterilize Muslim girls [21] and those that circulated in Pakistan in 2011 that the USA and its allies were running spying networks through vaccination campaigns [22] have contributed to a loss of community confidence in the immunization program. A series of fatal attacks in December 2012 and February 2013 targeting polio vaccination workers in Pakistan and Nigeria, respectively, has led to fear and confusion around vaccination campaigns and appears to have compromised the vaccine coverage in some areas. This continues to affect immunization uptake and intensive efforts have been made to engage local community and religious leaders to champion the cause.

The combination of missed targets for eradication and the high costs of implementing the GPEI's activities worldwide has prompted some public health officials to question the concept of eradication in favor of a strategy of "effective control". They argue that 
maintaining less than 500 polio cases per year would be cheaper than completing eradication [23]. This suggestion has so far been rejected by the international public health and donor communities, and continued polio surveillance still requires extensive financial and operational efforts. Epidemiological modeling has suggested that in low-income countries alone, a switch to 'control' would result in an estimated 4 million polio-paralyzed children over the next 20 years [24]. Furthermore, a 2010 study based on GPEI activities from 1988 through 2035 estimated that the elimination of wild poliovirus by 2015 would produce net profits of around US $\$ 50$ billion from reduced treatment costs and gains in economic productivity by preventing poliorelated disability, with low-income countries accounting for approximately $85 \%$ of the net benefits [25].

The gains from the global implementation of polio eradication initiatives are not only monetary. The GPEI has trained an enormous cadre of staff who understand basic health care needs and can provide services to people in the poorest areas in the world. Activities undertaken under the auspices of the GPEI have also contributed to the improvement of public health at large and increased the effectiveness of other preventive programs. Polio program staff have supported the surveillance of and response to measles, tetanus, meningitis, yellow fever and cholera. Furthermore, in many countries, the GPEI successfully expanded its delivery model to include the distribution of Vitamin A supplements alongside polio immunizations, estimated to have averted at least 1.1 million Vitamin A deficiency-related deaths from 1988 to 2010 [25].

In 2012, the World Health Assembly requested a comprehensive polio endgame strategy [26], which culminated in the development of the Polio Eradication and Endgame Strategic Plan 2013-2018 [27]. The Plan is based on broad consultations with national health authorities, global health initiatives, scientific experts, donor partners and other stakeholders. The Plan has four main objectives: to stop all wild poliovirus transmission by the end of 2014 and new cVDPV outbreaks within 120 days of confirmation of the first case; to strengthen immunization systems, introduce IPV into the routine immunization schedule globally and withdraw the use of oral polio vaccines; certify all regions of the world polio-free by 2018 and ensure the safe containment of all poliovirus stocks; and to ensure that the world remains permanently polio-free with careful legacy planning as well as planning for the transition of assets and the infrastructure of the polio program to benefit other development goals and global health interventions.

The Plan aims to withdraw the use of the type-2 component of OPV in all routine immunization programs by mid-2016. The importance of withdrawing the type-2 component as quickly as possible was reinforced by the 2012 polio outbreaks caused by circulating type-2 vaccine-derived polioviruses, which left 65 children paralyzed in 7 countries: Afghanistan, Chad, the Democratic Republic of Congo, Kenya, Nigeria, Pakistan and Somalia [28]. As of August 13, 2013, 17 cases of polio due to circulating type- 2 vaccine-derived polioviruses were reported in 6 countries: Afghanistan, Cameroon, Chad, Nigeria, Pakistan and Somalia [29].

The withdrawal of the type- 2 component of OPV will require the strengthening of immunization systems, the introduction of at least one dose of affordable IPV into the routine immunization schedule globally and then the 
replacement of tOPV with bOPV. This would pave the way for the eventual withdrawal of bOPV use in 2019-2020.

The GPEI is currently undertaking research to find ways of enabling low-income countries to access low-cost IPV options, instead of relying on costly imports. A multi-pronged research agenda is being pursued to investigate: a dose-reduction strategy using intradermal administration of fractional IPV doses; a schedule requiring fewer doses; adjuvant use to reduce the quantity of antigen required in the vaccine; and IPV production processes to facilitate manufacture in low-cost sites. The GPEI is also investigating the mucosal immune responses stimulated by IPV compared with those stimulated by OPV. In addition, work is being carried out to develop an IPV based on 'Sabin' attenuated virus seed-strains [30]. While traditional manufacturing of IPV involves large amounts of infectious 'Salk' seed strains, IPV containing the attenuated Sabin seed strains would reduce the severity of potential consequences in the event of a biocontainment failure at an IPV manufacturing facility.

Financing of the eradication effort remains a huge challenge. In the first quarter of 2012, GPEI activities were scaled down in 24 high-risk countries because of an acute funding shortage [31]. The budget for the Plan is US $\$ 5.5$ billion, with a peak spending in 2013, then estimated to decline annually [32]. As of June 1, 2013, the GPEI was tracking over US\$ 217 million in firm prospects, which if fully operationalized could close the 2013 funding gap, provided enough unspecified funds are secured to cover all cost categories [32]. However, pledges are very different to signed agreements and cash disbursements, and there is still a US \$1.5 billion funding gap to fully resource the Plan.
This shortfall has the potential to hamper the goal of eradication.

Today, eradication efforts continue. In 2012, 223 wild poliovirus cases were reported globally, more than a $60 \%$ decline compared with 2011 and only 5 countries reported cases in 2012 compared with 16 in 2011 [33]. As of August 13, 2013, 181 wild poliovirus cases had already been reported [33].

\section{CONCLUSION}

The global health effort to eradicate polio has faced numerous challenges since the launch of the GPEI. It is hoped that the last remaining obstacles have been identified and will be overcome within the established timeframe of the Polio Eradication and Endgame Strategic Plan. Crucially, success in the polio endgame would provide a strong evidence base and encourage political commitment to other such eradication initiatives. However, building on the lessons learned from the polio experience, any eventual strategy for measles eradication should strengthen routine immunization and not merely become a substitute [34].

\section{ACKNOWLEDGMENTS}

No funding or sponsorship was received for this study or publication of this article. Ms Lien is the guarantor for this article, and takes responsibility for the integrity of the work as a whole.

Conflict of interest. Gemma Lien and David L. Heymann declare no conflicts of interest.

Open Access. This article is distributed under the terms of the Creative Commons Attribution Noncommercial License which 
permits any noncommercial use, distribution, and reproduction in any medium, provided the original author(s) and the source are credited.

\section{REFERENCES}

1. Aylward B, Tangermann R. The global polio eradication initiative: lessons learned and prospects for success. Vaccine. 2011;29:D80-5.

2. Polio and Prevention, Global Polio Eradication Initiative 2013. http://www.polioeradication.org/ Polioandprevention.aspx. Accessed 19 August 2013.

3. Bunimovich-Mendrazitsky S, Stone L. Modeling polio as a disease of development. J Theor Biol. 2005;237:302-15.

4. History of Polio, Global Polio Eradication Initiative 2013. http://www.polioeradication.org/Polioand prevention/Historyofpolio.aspx. Accessed 30 August 2013.

5. Resolution No. WHA41.28: Global eradication of poliomyelitis by the year 2000. Forty-first World Health Assembly. World Health Organization 1988. http://www.who.int/ihr/polioresolution4128en.pdf. Accessed 19 August 2013.

6. About Us, Global Polio Eradication Initiative 2013. http://www.polioeradication.org/AboutUs.aspx. Accessed 30 August 2013.

7. Oral polio vaccine (OPV), Global Polio Eradication Initiative 2013. http://www.polioeradication.org/ Polioandprevention/Thevaccines/Oralpoliovaccine OPV.aspx. Accessed 19 August 2013.

8. Grassly N, Wenger J, Durrani S, Bahl S, Deshpande J, Sutter R, et al. Protective efficacy of a monovalent oral type 1 poliovirus vaccine: a case-control study. Lancet. 2007;369:1356-62.

9. Sutter R, John T, Jain H, Agarkhedkar S, Ramanan P, Verma $\mathrm{H}$, et al. Immunogenicity of bivalent types 1 and 3 oral poliovirus vaccine: a randomized, double-blind, controlled trial. Lancet. 2010;376:1682-8.

10. Heymann D, Sutter R, Aylward B. A vision of a world without polio: the OPV cessation strategy. Biologicals. 2006;34:75-9.

11. Inactivated polio vaccine (IPV), Global Polio Eradication Initiative 2013. http://www.polioeradication. org/Polioandprevention/Thevaccines/Inactivated poliovaccine(IPV).aspx. Accessed 30 August 2013.
12. Report of the Independent Monitoring Board of the Global Polio Eradication Initiative, April 2011. http:// www.polioeradication.org/Portals/0/Document/ Data\&Monitoring/IMB_Reports/IMB_Report_April 2011.pdf. Accessed 19 August 2013.

13. Aylward B, Acharya A, England S, Agocs M, Linkins J. Global health goals: lessons from the worldwide effort to eradicate poliomyelitis. Lancet. 2003;362:909-14.

14. Executive Board document EB107/28. Eradication of poliomyelitis: Report by the Secretariat. World Health Organization 2000. http://apps.who.int/gb/ archive/pdf_files/EB107/ee28.pdf. Accessed 19 August $201 \overline{3}$.

15. Executive Board document EB111/32. Eradication of poliomyelitis: Report by the Secretariat. World Health Organization 2002. http://apps.who.int/gb/ archive/pdf_files/EB111/eeb11132.pdf. Accessed 19 August 2013.

16. Transmission of wild poliovirus type 2: apparent global interruption. Wkly Epidemiol Record. 2001;76(13):95-97.

17. Grassly N, Fraser C, Wenger J, Deshpande J, Sutter $\mathrm{R}$, Heymann D, et al. New strategies for the elimination of polio from India. Science. 2006;314:1150-3.

18. Global Polio Eradication Initiative Annual Report 2009, World Health Organization 2010. http:// www.polioeradication.org/content/publications/ AnnualReport2009_ENG.pdf. Accessed 19 August 2013.

19. John T, Vashishtha V. Eradicating poliomyelitis: India's journey from hyperendemic to polio-free status. Indian J Med Res. 2013;137(5):881-94.

20. The Vaccines, Global Polio Eradication Initiative 2013. http://www.polioeradication.org/Polioand prevention/Thevaccines.aspx. Accessed 30 August 2013.

21. Yahya M. Polio vaccines-difficult to swallow. The story of a controversy in northern Nigeria. Institute of Development Studies. 2006; Working Paper 261. http://www.ids.ac.uk/files/Wp261.pdf. Accessed 19 August 2013.

22. Boone J. Taliban leader bans polio vaccinations in protest at drone strikes. The Guardian, 26 June 2012. http://www.guardian.co.uk/world/2012/jun/ 26/taliban-bans-polio-vaccinations. Accessed 19 August 2013.

23. The Case for Completing Polio Eradication, World Health Organization 2007. http://www.who.int/ 
immunization/sage/TheCase_FINAL.pdf. Accessed 19 August 2013.

24. Thompson K, Duintjer Tebbens R. Eradication versus control for poliomyelitis: an economic analysis. Lancet. 2007;369 (9570):1363-71.

25. Duintjer Tebbens R, Pallansch M, Cochi S, Wassilak S, Linkins J, Sutter R, et al. Economic analysis of the global polio eradication initiative. Vaccine. 2011;29(2):334-43.

26. Resolution No. WHA65.5: Poliomyelitis: intensification of the global eradication initiative. Sixty-fifth World Health Assembly. World Health Organization 2012. http://apps.who.int/gb/ebwha/ pdf_files/WHA65/A65_R5-en.pdf. Accessed 19 August 2013.

27. Polio Eradication and Endgame Strategic Plan 2013-2018: Executive Summary. Global Polio Eradication Initiative 2013. http://www. polioeradication.org/Portals/0/Document/ Resources/StrategyWork/PEESP_ES_EN_A4.pdf. Accessed 19 August 2013.

28. Poliomyelitis: intensification of the global eradication initiative, report by the Secretariat. Sixty-sixth World Health Assembly, document A66/18. World Health Organization 2013. http:// apps.who.int/gb/ebwha/pdf_files/WHA66/A66_18en.pdf. Accessed 19 August 2013.

29. Circulating vaccine-derived poliovirus (cVDPV) 2000-2013 (data as of 13 August 2013), Global
Polio Eradication Initiative 2013. http://www. polioeradication.org/Dataandmonitoring/Polio thisweek/Circulatingvaccinederivedpoliovirus.aspx. Accessed 19 August 2013.

30. Polio vaccine technology transfer continues, 20 March 2013, Global Polio Eradication Initiative. http://www.polioeradication.org/Mediaroom/News stories/Newsstories2013/tabid/488/iid/286/Default. aspx. Accessed 19 August 2013.

31. Global Polio Eradication Initiative Annual Report 2011, World Health Organization 2012. http:// www.polioeradication.org/Portals/0/Document/ AnnualReport/AR2011/GPEI_AR2011_A4_EN.pdf. Accessed 19 August 2013.

32. Financial Resource Requirements 2013-2018: as of 1 June 2013, World Health Organization 2013. http:// www.polioeradication.org/Portals/0/Document/ Financing/FRR_EN_A4.pdf. Accessed 19 August 2013.

33. Polio this week-as of 13 August 2013, Global Polio Eradication Initiative, 2013. http://www. polioeradication.org/Dataandmonitoring/Poliothis week.aspx. Accessed 19 August 2013.

34. Heymann D, Fine P, Griffiths U, Hall A, MounierJack S. Measles eradication: past is prologue. Lancet. 2010;376:1719. 